\title{
Cost-Efficient Wireless Mobile Backhaul Topologies: An Analytical Study
}

\author{
Fang-Chun Kuo, Frank A. Zdarsky, Johannes Lessmann and Stefan Schmid \\ NEC Network Laboratories, NEC Europe Ltd., U.K. \\ Email: \{kuo, zdarsky, lessmann, schmid\}@neclab.eu
}

\begin{abstract}
Wireless communication technologies such as microwave radios are used to provide high-speed mobile backhaul connectivity for radio access networks in cases in which wirebased alternatives, e.g. cable or fiber, are not readily available and cannot be deployed in an economic or timely manner. Current mobile backhauls are predominantly deployed in tree or ring topologies, which simplify traffic management. Yet, with the increasing demand on backhaul capacity and the immense cost pressure on mobile backhaul solutions, meshed wireless mobile backhauls have been identified as a promising evolution. While traffic management in wireless mesh networks have been studied extensively in the literature, so far there is no quantitative analysis comparing the different topology options, i.e. mesh, ring and tree, regarding network performance and deployment cost. This paper fills this gap by studying the minimum cost problem of connecting a set of base station/gateway sites using different topologies while supporting both time- and space-varying traffic demands. Furthermore, we consider the additional constraint of resilience to single link failures. The evaluation results show that meshed wireless backhaul topologies are a cost-effective alternative to trees and rings, in particular in the face of spatial and temporal fluctuation of traffic demand and protection against link failures.
\end{abstract}

\section{INTRODUCTION}

Due to the rapid growth of mobile data and emergence of newer bandwidth-intensive applications, the bandwidth demand of mobile users is continuing to rise. The backhaul in the radio access network (RAN), i.e. the network between the point of mobile access and the core of the network, is evolving to accommodate this growing demand and to prepare for the next generation of wireless cellular networks, such as LTE and LTE-Advanced.

To this date, most of the present backhaul networks use T1/E1 leased lines. This time division multiplexing (TDM)based backhaul was designed for voice networks requiring fixed bandwidths per call, and will not be able to sustain the high bandwidth demands of the next generation of mobile networks. Some options providing high-capacity backhaul transport are cable, fiber or wireless. Building backhaul networks using traditional copper cable or fiber network connections can take a lot of time and can be prohibitively expensive. On the other hand, using emerging wireless technologies, e.g. pointto-point microwave or free space optics (FSO), for backhauling has been studied in recent literature [2], [3], [4]. They are now capable of transmitting more than $1.25 \mathrm{~Gb} / \mathrm{s}$, and can bridge long distances between a gateway and a base station ${ }^{1}$.

\footnotetext{
${ }^{1}$ For generalization, in this paper we use base stations to represent base transceiver stations (BTSs) in 2G, NodeBs (NBs) in 3G or and evolved NodeBs (eNBs) in LTE. Similarly, we use gateways to represent base station controllers (BSCs) in $2 \mathrm{G}$ networks, the radio network controllers (RNCs) in $3 \mathrm{G}$ networks and serving gateways (SGWs) in LTE networks.
}

In this context, backhaul networks using wireless technologies, namely wireless mobile backhauls, are a good complement to wired (optical or copper) based backhauls due to their high data rates, ease of installation and scalability [1].

Aside from applying new transmission technologies in RANs, the design of topologies for mobile backhaul has to be re-visited for the sake of providing carrier-class reliability at low system cost. As shown in Figure 1, the present backhaul architecture for RANs are typically based on tree topologies interconnecting base stations and gateways. This tree topology is very sensitive to any kind of failures, e.g. line of sight blockage in wireless links or random device failure. Although the low reliability of tree topologies can be improved by using $1+1$ link protection, deploying 1+1 link protection for every link is very costly. Since mobile operators have estimated that 25-50 percent of their operating costs are associated with the backhaul [5], any strategy that is developed to enhance the network performance must be cost-effective.

In this paper we focus on designing a cost-effective wireless mobile backhaul for RANs. We assume that base station sites are given (as a result from radio access planning) and that the wireless backhaul operates on a different frequency band than the access. Our problem is thus different from that of optimizing radio relay deployments. We further assume that nodes are equipped with multiple radios and directional antennas. These point-to-point wireless links are set up by high-gain directional antennas mounted on top of towers to achieve line-of-sight (LOS). In the domain of optimization of mobile backhaul networks with point-to-point wireless links, the benefits of mesh-based networks have been recently explored [11], [8], [9], [10], [12], [7], which include reliability to link failures and robustness to traffic fluctuations owing to the provisioning of multiple paths. In [6], a mesh-based restorable topology has been designed for survivable backhaul. However, there is so far no quantitative analysis to compare mesh topologies with other topologies, e.g. ring or tree topologies, in the aspect of performance and deployment costs for wireless mobile backhaul networks. Therefore, the goal of this paper is to study the deployment issues of planning different topologies to minimize system cost.

More specifically, we tackle the following question: given a network layout (the locations of the base stations and gateways), what is the optimal topology for the mobile backhaul with point-to-point wireless links that minimizes the deployment cost while meeting the expected traffic demands for all base stations? Our main contributions are outlined below: 
- We first formulate the minimum cost problem of connecting base stations and gateways using different topologies under the constraint of system robustness towards varying traffic demands over time and space.

- We further address the issue of topology design in terms of resilience to a single concurrent link failure.

Our work adds to the understanding of the topology impact for wireless mobile backhaul network deployment and their general impact on performance.

The rest of the paper is structured as follows. In Section II, the problem statement and the model of the topology planning problem are introduced. In Section III, the problem of planning a primary topology with the constraint of traffic fluctuations robustness is formulated. Furthermore, the backup topology planning problem under the single link failure constraint is formulated in Section IV. In Section V, the topology planning problems with traffic fluctuations and single failure model are evaluated. Finally, we present our conclusions in Section VI.

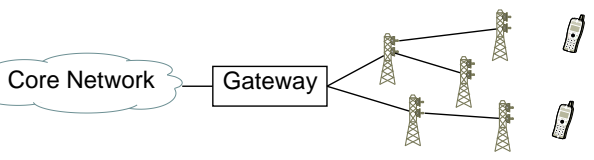

Fig. 1. Current radio access network

\section{Problem Statement and Model}

Topology choices for wireless mobile backhaul networks are trees, rings or meshes. Selecting the topology can prove to be a complicated task. Network planners should consider the particular environmental conditions, business conditions (such as spectrum, radio and antennas costs), reliability requirements, and application characteristics, in order to determine the best solution for their needs. In this paper, we address the question: Which type of topology is the most cost-effective under different requirements of traffic demand and reliability?

Unlike deploying wired links, e.g. cable or fiber, the cost of wireless links does not primarily correlate with the distance but corresponds to the fixed cost of installing a transmitter/receiver at both ends of the links. In other words, the dominant cost for deploying wireless mobile backhaul networks is the set of wireless links. Therefore, our optimization objective can thus be stated as: Find the tree, ring and mesh topology, respectively, with the minimum cost of wireless link setup, given a set of base station/gateway sites.

While minimizing the cost, we also wish to achieve a certain desired level of network performance. We formulate this as constraints in our topology-planning problem. In this paper, we qualify performance simply by saying that each base station requires a certain traffic demand to be met towards the gateways. In the problem formulation, we denote this traffic demand requirement by $t_{m}$ for the base station $m$ and term the constraint as the traffic constraint. Another network performance is qualified by measuring the capability of resilience. This constraint, termed as the resilience constraint, is formulated by ensuring the traffic demand requirement is not compromised when any single link failure occurs.
TABLE I

NOTATIONS

\begin{tabular}{|r|l|}
\hline$G$ & A set of gateways in the network, $G \subset V$. \\
\hline$M$ & A set of base stations in the network, $M \subset V$. \\
\hline $\mathbb{A}$ & $\begin{array}{l}\text { A primary topology } \mathbb{A}:\left\{\alpha_{i, j} \mid(i, j) \in E\right\}, \text { where } \alpha_{i, j}=1 \text { if a } \\
\text { primary wireless link }(i, j) \text { is deployed, and } 0 \text { otherwise. }\end{array}$ \\
\hline $\mathbb{B}$ & $\begin{array}{l}\text { A backup topology } \mathbb{B}:\left\{\beta_{i, j} \mid(i, j) \in E\right\}, \text { where } \beta_{i, j}=1 \text { if a } \\
\text { backup wireless link }(i, j) \text { is deployed, and } 0 \text { otherwise. }\end{array}$ \\
\hline $\mathbb{T}$ & $\begin{array}{l}\text { Traffic profile representing a set of traffic demands required by the } \\
\text { base stations in the network. } \mathbb{T}:\left\{t_{m} \mid m \in M\right\} .\end{array}$ \\
\hline $\mathfrak{T}$ & $\begin{array}{l}\text { Multiple traffic profiles representing traffic fluctuations over time } \\
\text { and locations. }\end{array}$ \\
\hline$c_{i, j}$ & Link capacity of the edge $(i, j) \in E$. \\
\hline$f_{i, j}$ & Flow traffic of the edge $(i, j) \in E$. \\
\hline$x_{i, j}$ & $\begin{array}{l}\text { The cost associated to install a primary wireless link in the edge } \\
(i, j) \in E .\end{array}$ \\
\hline$y_{i, j}$ & $\begin{array}{l}\text { The cost associated to install a backup wireless link in the edge } \\
(i, j) \in E .\end{array}$ \\
\hline
\end{tabular}

For convenience, all notations are listed in Table I. Our problem can be formulated using a graph theoretic approach. Given a network $N=(V, E)$ to be deployed for wireless mobile backhaul networks, the set $V$ and $E$ represents the vertices and edges in the network $N$, respectively. A set $G \subset$ $V$ is determined as gateways. A set $M \subset V$ is determined as base stations. The capacity of the wireless link for the edge $(i, j)$ is denoted as $c_{i, j},(i, j) \in E$. A traffic profile represents a set of traffic demands required by base stations, i.e. $\mathbb{T}$ : $\left\{t_{m} \mid m \in M\right\}$, where $t_{m}$ is the traffic demand for the base station $m$ to gateways. To account for fluctuations in network traffic over time and locations, we consider multiple traffic profiles $\mathfrak{T}:\left\{\mathbb{T}_{1} \mathbb{T}_{2} \ldots\right\}$. By using multiple traffic profiles as the traffic constraint, the topology is ensured to tolerate traffic fluctuations.

The point-to-point wireless links are modeled as two types, namely primary and backup links. A primary topology is specified by a set of values $\mathbb{A}:\left\{\alpha_{i, j} \mid(i, j) \in E\right\}$, where $\alpha_{i, j}=1$ if a primary wireless link $(i, j)$ is deployed, and 0 otherwise. Similarly, a backup topology $\mathbb{B}:\left\{\beta_{i, j} \mid(i, j) \in E\right\}$, where $\beta_{i, j}=1$ if a backup wireless link $(i, j)$ is deployed, and 0 otherwise. Each edge $(i, j) \in E$ is associated with a cost $x_{i, j}$ for setting up a primary wireless link, while the additional cost required to install a backup link for edge $(i, j) \in E$ is denoted as $y_{i, j}$. In our analysis, we first consider the problem of planning a primary topology with the constraint of traffic fluctuations robustness in Section III. Finally, the backup topology planning problem is addressed to be resilient to any single link failure in Section IV.

\section{TRAFFiC Fluctuation Robust Topologies}

In this section, we study the problem of determining a minimum cost topology robust to traffic fluctuations in wireless mobile backhaul networks. To account for traffic fluctuations over time and locations, a set of multiple traffic profiles $\mathfrak{T}$ is considered. Given a set of link capacities and link costs, our primary topology planning problem for traffic fluctuation robustness can be formulated as follows:

$$
\min _{\mathbb{A}} \sum_{(i, j) \in E} x_{i, j} \alpha_{i, j}
$$


subject to

$$
\begin{gathered}
0 \leq f_{i, j} \leq c_{i, j} \alpha_{i, j}, \forall(i, j) \in E \\
\sum_{(i, g) \in E, g \in G} f_{i, g}=\sum_{\mathbb{T}} t_{m}, \forall \mathbb{T} \in \mathfrak{T}
\end{gathered}
$$

$t_{m}+\sum_{j:(j, m) \in E, m \in M} f_{j, m}=\sum_{i:(m, i) \in E, m \in M} f_{m, i}, \forall t_{m} \in \mathbb{T}, \mathbb{T} \in \mathfrak{T}$

where $f_{i, j}$ is the flow traffic of the edge $(i, j) \in E$.

The objective function (1) accounts for the total cost of the network, i.e. installing primary wireless links. Constraint (2) assures that the flow traffic cannot exceed the edge capacity. Constraints (3) and (4) define that, given a topology, the overall flow traffic passing through the gateways equals the overall traffic demand, i.e. the traffic profile has to be satisfied. For simplicity, we will denote the combination of constraints (2), (3) and (4) by $T D C(\mathbb{A}, \mathfrak{T})$, representing the traffic demand constraint for a given topology $\mathbb{A}$ and traffic profile set $\mathfrak{T}$. We say that a topology $\mathbb{A}$ is feasible with a given set of traffic profiles $\mathfrak{T}$ iff the $T D C(\mathbb{A}, \mathfrak{T})$ can be satisfied.

For checking the feasibility of a topology, i.e. whether the requirement of multiple traffic profiles is fulfilled, we solve a max-flow problem per traffic profile. Max-flow problems are easily solved for the single-source, single-sink case using an Edmunds-Karp algorithm. The present case of multiple base stations and gateways can be equally solved by adding a virtual source $s$ and sink as shown in Figure 2. The virtual source connects to all the base stations $m$ with the capacity of the required traffic demands $t_{m}$ on each edge while the virtual sink $t$ connects to all the gateways $g$ with infinite capacity on each edge. If the calculated maximum flow is greater than the sum of the required traffic demands in the network, this topology is then identified as a feasible topology.

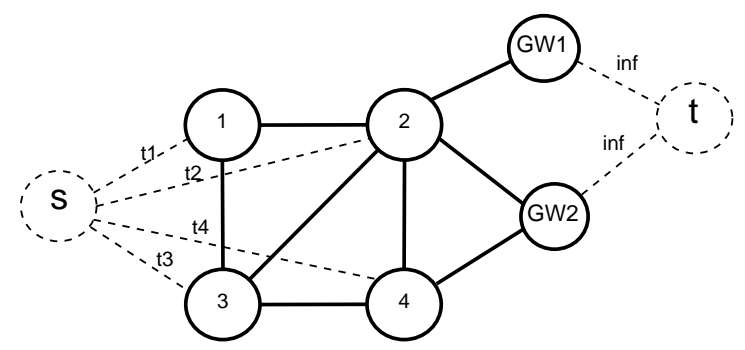

Fig. 2. Transformation of a topology feasibility checking problem into a maximum flow problem.

\section{Failure Resistant Topologies}

For deploying a link failure resistant topology, redundant backup links are supplied to maintain service continuity in the presence of failures. Therefore, a failure resistant topology includes not only a primary topology $\left.\mathbb{A}:\left\{\alpha_{(} i, j\right) \mid(i, j) \in E\right\}$ but also a backup topology $\left.\mathbb{B}:\left\{\beta_{i} i, j\right) \mid(i, j) \in E\right\}$. We adopt the single link failure model, which means we assume only one link failure occurs at a time. We define a primary topology $\mathbb{A}$ with a faulty link $(\hat{i}, \hat{j})$ as $\mathbb{A}^{\prime}(\hat{i}, \hat{j}):\left\{\alpha_{i, j}^{\prime} \mid(i, j) \in E\right\}$, where

$$
\alpha_{i, j}^{\prime}= \begin{cases}0 & \text { if }(i, j)=(\hat{i}, \hat{j}) \\ \alpha_{i, j} & \text { otherwise }\end{cases}
$$

Under the single link failure model, a backup link is set up for the faulty link $(\hat{i}, \hat{j})$ if the primary topology with that faulty link $\mathbb{A}^{\prime}(\hat{i}, \hat{j})$ is not feasible to accommodate the multiple traffic profiles $\mathfrak{T}$.

$$
\beta_{\hat{i}, \hat{j}}= \begin{cases}1 & \text { if } \operatorname{TDC}\left(\mathbb{A}^{\prime}(\hat{i}, \hat{j}), \mathfrak{T}\right) \text { is not feasible } \\ 0 & \text { otherwise }\end{cases}
$$

We denote this constraint as $S F C(\mathbb{B}, \mathbb{A}, \mathfrak{T})$ representing single failure constraint.

The backup topology planning problem with single link failure model is thus formulated as:

$$
\min _{\mathbb{A}, \mathbb{B}} \sum_{(i, j) \in E} x_{i, j} \alpha_{i, j}+y_{i, j} \beta_{i, j}
$$

subject to $T D C(\mathbb{A}, \mathfrak{T}), S F C(\mathbb{B}, \mathbb{A}, \mathfrak{T})$.

The objective function (6) accounts for the total cost of the network including installing primary links and backup links. Two constraints are considered for the primary topology and the backup topology. First, the traffic demand constraint $T D C(\mathbb{A}, \mathfrak{T})$ should be fulfilled with the primary topology $\mathbb{A}$ when there is no failure. Second, under the single failure constraint $\operatorname{SFC}(\mathbb{B}, \mathbb{A}, \mathfrak{T})$, the primary topology $\mathbb{A}$ together with the backup topology $\mathbb{B}$ should satisfy the traffic demand requirement despite any single link failures.

\section{Evaluation}

In this section, we evaluate the deployment problem with tree, ring and mesh topologies. We first introduce our parameter settings. Finally, the topology planning problem with the constraints of traffic fluctuation robustness and failure resilience are analyzed.

\section{A. Parameter Setting}

For generating random geographic node locations with a given node density, nodes are placed based on a random uniform distribution with the following constraints: In the generation of the node locations, each node is required to have at least one node to communicate, i.e. there is at least one node within its communication range. Another constraint for node placement is that the distance between any two nodes must be greater than $1 \mathrm{~km}$. The node density is 0.5 nodes $/ \mathrm{km}^{2}$. We assume that all links achieve line-of-sight (LOS), i.e. no obstructions, in our simulation. Thus, in the communication connectivity graph model we assume that any node pair can communicate iff their Euclidean distance is within a communication radius. The transmission distance varies with the type of equipment that is used, such as radio card, antenna type and its power. In our evaluation, the communication radius is set to $10 \mathrm{~km}$. The link capacity is $156 \mathrm{Mbps}$ if the distance is within the communication radius $10 \mathrm{~km}$ and 0 otherwise.

We evaluate the deployment problem using integer linear programming (ILP). Tree and ring topologies are produced by adding constraints on the loop-freeness and number of incident edges, respectively, to the ILP. However, the presented integer program can be solved in a reasonable time (days) only for small size problems, i.e. small networks. For our 
evaluation, we use networks of 15 nodes. However, this should be fully sufficient for the following reasons. First, typical wireless mobile backhaul networks are normally not much bigger than 15 nodes. Second, a wireless network with 15 nodes is structurally (i.e. in terms of node density, node degree, node distribution) equivalent to a larger network due to the limited wireless transmission ranges. Hence, we believe that our results may be likewise applicable to larger networks.

The deployment cost of the wireless mobile backhaul network is mainly the sum of the cost of setting up each of the wireless links. Each wireless link generally incurs a fixed cost for setting up the transmitter/receiver pair. In other words, the cost of the wireless mobile backhaul network is mainly determined by how many wireless links are needed to be set up. Therefore, in our evaluation we consider the problem of finding topologies with the minimum number of links that can achieve the given demands. Furthermore, we assume that adding parallel links between two nodes is not feasible due to cross-link interference caused by limited frequency resources.

For modeling traffic fluctuations over different areas, we color the base stations into two groups $\zeta_{1} \subset M$ and $\zeta_{2} \subset M$ ( $\zeta_{1} \cup \zeta_{2}=M, \zeta_{1} \cap \zeta_{2}=\emptyset$ ) according to their locations. Intuitively, this could be a separation between commercial and residential areas, for example. We define the traffic fluctuation between two areas with a given traffic profile $\mathbb{T}$ as

$$
\Delta\left(\mathbb{T}, \zeta_{1}, \zeta_{2}\right)=\left|\frac{\sum_{m \in \zeta_{1}} t_{m}-\sum_{m \in \zeta_{2}} t_{m}}{\sum_{m \in M} t_{m}}\right| .
$$

Traffic demands may fluctuate over time and locations. To account for this, we consider multiple traffic profiles $\mathfrak{T}$ in which the traffic demand $t_{m}$ is randomly generated, the overall traffic demand in the network is $\sum_{t_{m} \in \mathbb{T}} t_{m}=T$, and the traffic fluctuation between two areas is bounded by a certain upper limit, i.e. $\Delta\left(\mathbb{T}, \zeta_{1}, \zeta_{2}\right) \leq \eta, \forall \mathbb{T} \in \mathfrak{T}$ where $\eta$ is traffic fluctuation degree.

\section{B. Traffic Fluctuation Robustness}

We first study how the traffic affects the network topology planning. Figure 3 shows the number of links for the tree, ring and mesh topologies under four different traffic fluctuation degrees, $0 \%, 20 \%, 40 \%$ and $60 \%$. The following observations can be made:

1) The tree topology requires fixed number of links until the maximum feasible traffic demand is reached. Unlike the tree topology, the number of links in the ring topology increases when the total traffic demand increases. This is because smaller rings, which incur more links, can be created in order to accommodate a higher traffic demand.

2) Above a certain traffic demand threshold, the optimization problem using a tree or ring topology becomes infeasible. In other words, it is no longer feasible to fulfill the traffic demand constraint using a tree or ring topology with a given radio technology and frequency bandwidth. On the other hand, mesh topologies can accommodate more traffic demand by simply adding more links in the network.
3) When the traffic fluctuation degree increases, the maximum feasible traffic demand in the ring and tree topologies decreases. Therefore, the only way to improve the robustness to traffic fluctuations is to upgrade the link capacity in the tree and ring topology. Since the traffic peak could occur at almost any place in the network, this implies that most of the links need to be upgraded. However, the mesh topology can flexibly add few links so that traffic fluctuations over the locations can be balanced by routing the traffic via the redundant links to avoid congestion.

In summary, while upgrading link capacities to accommodate localized peak traffic in tree or ring topologies is an expensive endeavor, mesh topologies provide the flexibility to install incremental additions of links in order to deal with fluctuating peak traffic in the network.
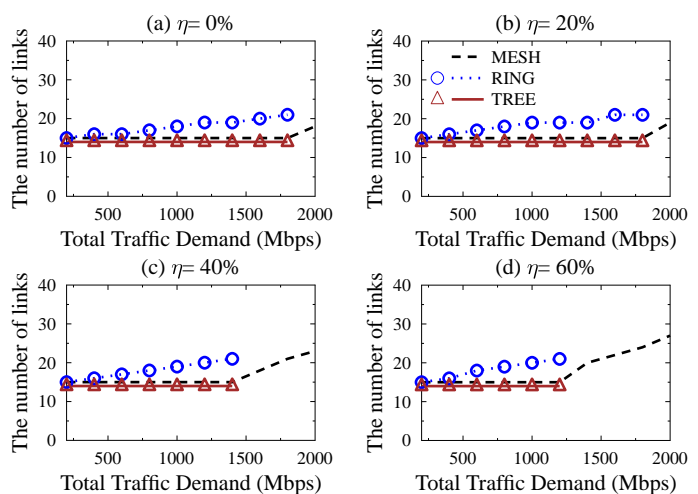

Fig. 3. Number of links (objective value) under different traffic fluctuation degrees in mesh, ring and tree topologies

\section{Failure Resilience}

In this section we study the topology planning problem under the constraint of link failure resilience. The results are shown in Figure 4. Furthermore, we use link ratio as a metric to evaluate the ratio of additional links deployed for supporting failure resilience. Hence, we define the link ratio as $\frac{n_{\text {resilience }}-n_{\text {normal }}}{n_{\text {normal }}}$ where $n_{\text {resilience }}$ and $n_{\text {normal }}$ are the number of links in the failure resilient topology and in the topology designed for no failure, respectively. The results are shown in Figure 5. Our observations are summarized below:

1) In the tree topology, all links should be protected by backup links even when the required traffic demand is low. Accordingly, the link ratio is $100 \%$ as shown in Figure 5. This leads to higher equipment and maintenance costs. Furthermore, although the protected tree topology is indeed protected against link failures, it does not provide any path redundancy. Therefore, the protected tree topology is still vulnerable to traffic fluctuations as shown in Figure 4.

2) In the ring topology, an alternate route is always available in case of link failure. Nevertheless, a failure in a ring results in halving the capacity in the ring in the worse case. Hence, a few extra primary links must be supplied to form smaller rings to achieve link failure protection without adding backup links. Accordingly, in Figure 5 the ring curves increase slowly with small traffic demand requirement. Furthermore, in Figure 5 the link ratio for the ring topology shows a big jump with higher 
traffic demand requirement. This is because when the size of rings cannot be reduced since the smallest size of a ring is 3 nodes. and the traffic demand is not yet satisfied, many backup links are then needed for each small rings to compensate the loss of network capacity due to the failure. To sum up, without upgrading the link capacity, the alternate routes provided by the ring topology do not help a lot to reduce the number of additionally required links for failure resilience.

3) As depicted in Figure 4, the mesh topology requires the least number of links for protecting against link failures when compared to the ring and tree topologies. Furthermore, the link ratio is low in the mesh topology. This is because few redundant links in the mesh can already yield high path diversity. Hence, when a failure happens in the network, the traffic can still be redirected to other paths without compromising the network performance. Note that the link ratio for mesh topologies drops with higher traffic demand. This is because, for accommodating this higher traffic demand, additional links have to be deployed even without failure resilience support as shown in Figure 3. Therefore, only few additional links are needed for link failure protection, which incurs lower link ratio.
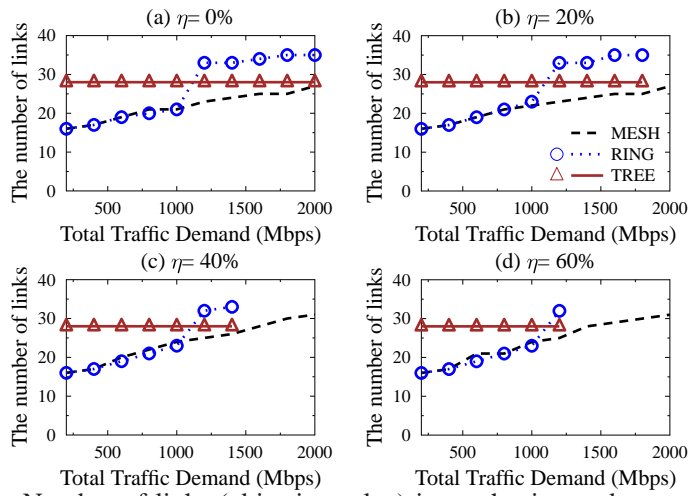

Fig. 4. Number of links (objective value) in mesh, ring and tree topologies with single link failure resilience support under different traffic fluctuation degrees.
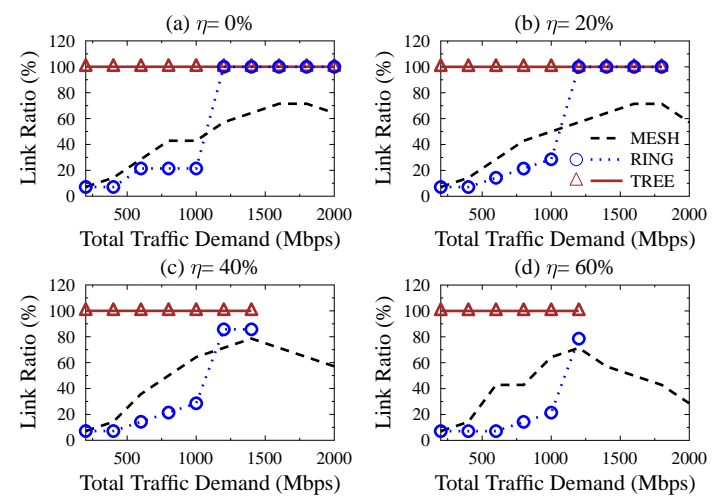

Fig. 5. Link ratio in mesh, ring and tree topologies under different traffic fluctuation degrees.

\section{CONCLUSION}

In this paper, we have provided a quantitative analysis comparing different topology options (mesh, tree, and ring) for wireless mobile backhauls in RANs in terms of network performance and deployment cost. The basis for this comparison has been formulated as a mathematical optimization problem that, for a given set of base station and gateway sites and given traffic demands, determines the cost-optimal mesh, tree, and ring topologies, respectively.

We first considered the effect of traffic fluctuations (e.g. due to natural day-time / night-time fluctuations between office and residential areas). We showed that traffic demands above a certain threshold can only be supported by mesh topologies, while tree and ring topologies reach their capacity limits. Furthermore, if the fluctuation of the traffic demand increases, the relative advantage of mesh topologies increases, as they allow traffic to be load-balanced over the topology to mitigate congestion.

Moreover, the optimization problem has then been extended to be resilient against any single link failures. Results show that mesh topologies can do so with fewer links than tree and ring topologies, while again supporting high traffic demands is not feasible in tree and ring topologies.

\section{ACKNOWLEDGMENT}

The research leading to these results has received funding from the European Community's Seventh Framework Programme (FP7/2007-2013) under grant agreement $n^{\circ} 214994$.

\section{REFERENCES}

[1] Paul A. Kennard, On the BackSwing, Telecommunications - Americas Edition, 2002.

[2] A. Acampora, S.H. Bloom and S. Krishnamurthy, UniNet: a hybrid approach for universal broadband access using small radio cells interconnected by free-space optical links, in IEEE Journal on Selected Areas in Communications, Volume 16, Issue 6, Pages 973-988; August 1998.

[3] David Falconer, A system architecture for broadband millimeter-wave access to an ATM LAN, IEEE Personal Communications, vol. 3, no. 4, Pages 36-41, August. 1996.

[4] V.R.M. Thyagarajan, R.H.M. Hafez and D.D. Falconer, Broadband indoor wireless communications in the (2060) GHz band: signal strength considerations, 2nd International Conference on Universal Personal Communications, Volume 2, Pages 894-899, October 1993.

[5] Dean M. Johnson, Mobile Backhaul Using $39 \mathrm{GHz}$ spectrum,First Avenue Networks, October 2003

[6] Wee-Seng Soh, Zoe Antoniou and Hyong S. Kim, H. Murata and S. Yoshida, Improving Restorability in Radio Access Networks, IEEE GLOBECOM, San Francisco, California, USA, Pages 3493-3497, December 2003.

[7] S. Ghosh, K. Basu and Sajal Das, Mesh-up: Self-organizing mesh-based topologies for next generation radio access networks, Ad Hoc Networks, Volume 5, Issue 6, Pages 652-679, August 2007.

[8] Y. Aburakawa and T. Otsu, Experimental evaluation of 800-nm band optical wireless link for new generation mobile radio access network, International Topical Meeting on Microwave Photonics, Pages 261-264, 2002.

[9] Y. Aburakawa, T. Otsu and Y. Yamao, Fiber and free-space hybrid optical networking for new generation mobile radio access network, in the 5th International Symposium on Wireless Personal Multimedia Communications, Volume 2, Pages 586-590, October 2002.

[10] V. Kukshya, T.S. Rappaport, H. Izadpanah, G.Tangonan, R.A Guerrero, J.K Mendoza and B. Lee, Free-space optics and high-speed RF for next generation networks propagation measurements, in Proceedings of IEEE 56th Vehicular Technology Conference. VTC 2002-Fall, Volume 1, Pages 616-620.

[11] Y. Yamao, H. Suda, N. Umeda and N. Nakajima, Radio access network design concept for the fourth generation mobile communication system, in Proceedings of IEEE Vehicular Technology Conference. VTC 2000Spring, Tokyo, Volume 3, Pages 2285-2289, May 2000.

[12] T. Bu, Mun Choon Chan, and Ramachandran Ramjee, Designing Wireless Radio Access Networks for Third Generation Cellular Networks, in Proceedings of IEEE INFOCOM, Volume 1, Pages 68-78, March 2005. 